PROCEEDINGS OF THE

AMERICAN MATHEMATICAL SOCIETY

Volume 139, Number 10, October 2011, Pages 3431-3443

S 0002-9939(2011)10785-8

Article electronically published on March 4, 2011

\title{
SUPER-ADDITIVE SEQUENCES AND ALGEBRAS OF POLYNOMIALS
}

\author{
KEITH JOHNSON
}

(Communicated by Irena Peeva)

\begin{abstract}
If $K$ is a field with discrete valuation $\nu$ and $D=\{a \in K: \nu(a) \geq$ $0\}$, then an algebra $D[x] \subseteq A \subseteq K[x]$ has associated to it a sequence of fractional ideals $\left\{\mathcal{I}_{n}: n=0,1,2, \ldots\right\}$ with $\mathcal{I}_{n}$ consisting of 0 and the leading coefficients of elements of $A$ of degree no more than $n$ and a sequence of integers $\{a(n): n=0,1,2, \ldots\}$ with $a(n)=-\nu\left(\mathcal{I}_{n}\right)$. Combinatorial properties of this integer sequence reflect algebraic properties of $A$, and these are used to identify the degrees of generators of $A$ and to characterize finitely generated algebras $A$ by a periodicity property of this sequence.
\end{abstract}

\section{INTRODUCTION}

Let $K$ be a field with discrete valuation $\nu: K^{*} \rightarrow \mathbb{Z}$ and $D=\{a \in K: \nu(a) \geq$ $0\}$ the corresponding valuation domain. Let $\mathcal{M}=\{a \in D: \nu(a)>0\}$ be the unique maximal ideal of $D$. If $D[x] \subseteq A \subseteq K[x]$ is a subalgebra, then there is associated to $A$ a nested sequence of fractional ideals $\left\{\mathcal{I}_{n}: n=0,1,2, \ldots\right\}$ called the characteristic ideals of $A$ with $\mathcal{I}_{n}$ consisting of 0 and the leading coefficients of elements of $A$ of degree no more than $n$. The characteristic sequence of $A$, given by $\{a(n): n=0,1,2, \ldots\}$ with $a(n)=-\nu\left(\mathcal{I}_{n}\right)$, encapsulates some of the algebraic structure of $A$. Because $A$ is an algebra, the $a(n)$ 's satisfy the inequality $a(n) \geq a(i)+a(j)$ for all $i, j \geq 0$ with $i+j=n$ and so are examples of super-additive sequences. The plan of this paper is to describe some combinatorial properties of such sequences and point out how these results reflect the structure of the algebra A.

A particular class of algebras of the sort described above are those defined by integrality conditions. If $E$ is a subset of $D$, then $\operatorname{Int}(E, D)=\{f \in K[x]: f(E) \subseteq D\}$ is called the algebra of integer valued polynomials on $E$. The study of such algebras has a long history going back at least to the work of Polya and Ostrowski ([12, [1]) in 1919 (see [6] for a systematic treatment and a comprehensive bibliography up to 1997). For algebras of this and related sorts the results of Bhargava ([1, 2]) describe the characteristic sequences of these algebras in terms of specific orderings of the set $E$, and this leads to a recursive method for computing their characteristic sequences in some cases $([8,4])$. The method involves decomposing the sequence as a shuffle or a merge of two or more subsequences. We will investigate

Received by the editors May 21, 2010 and, in revised form, August 30, 2010.

2010 Mathematics Subject Classification. Primary 13F20; Secondary 05A10, 11C08.

(C)2011 American Mathematical Society

Reverts to public domain 28 years from publication 
this construction for super-additive sequences generally and show that it gives a lower bound for the characteristic sequence of the intersection of two such algebras.

One question about algebras which is always of interest is that of determining a generating set. In the case of the algebras $\operatorname{Int}(E, D)$ for sets $E$ which are homogeneous, i.e. a finite union of cosets of some power of the ideal $\mathcal{M}$, it was shown in [5] that a finite generating set exists if and only if $|D / \mathcal{M}|=\infty$. The original motivation for the present paper was to find a method for computing a generating set in this case. Since then it has been shown (4) that a variant of $\operatorname{Int}(E, D)$, called the algebra of polynomials integer valued on $E$ of finite order $k, \operatorname{Int}_{k}(E, D)$, is finitely generated for all $E$, and our method applies in this case also.

In more detail the paper is organized as follows. In section 2 we develop the basic properties of super-additive sequences, define the shuffle product and show that the shuffle product of super-additive sequences is super-additive. This is accomplished by relating super-additive sequences both to sub-additive sequences and to the classes of super and sub-additive functions. The latter, which has many applications, has a substantial literature, and we establish our results by appealing to known results in this area. (A curious feature of this development is that there is a product for functions analogous to the shuffle product of sequences which also preserves super-additivity but which seems not to have been previously studied.) In section 3 we relate generators for $A$ to points at which the super-additive sequence is strictly super-additive, i.e. $n$ for which $a(n)>a(i)+a(j)$ for all $i, j>0$ with $i+j=n$. The proof in [4 that $\operatorname{Int}_{h}(D, E)$ is finitely generated depends on showing that the corresponding characteristic sequence has the periodicity property that there exist $h, q$ such that $a(n+q)=a(n)+h$ for all $n \geq 0$ and that this property is preserved by the shuffle product. In general, it is true that a characteristic sequence having this property implies that the corresponding algebra is finitely generated; however the converse does not hold. Instead we show in section 4 that characteristic sequences of finitely generated algebras have the asymptotic periodicity property that beyond some point they agree with a super-additive sequence satisfying the condition above and that this characterizes them.

\section{Super-ADditive SEQUenCES AND The Shuffle PROdUCT}

Definition 2.1. (i) A nondecreasing sequence $\{a(n): n=0,1,2, \ldots\}$ of nonnegative integers with $a(0)=0$ is super-additive if $a(i+j) \geq a(i)+a(j)$ for all $i, j \geq 0$ and sub-additive if $a(i+j) \leq a(i)+a(j)$ for all $i, j \geq 0$.

(ii) A nondecreasing function $f: \mathbb{R}^{\geq 0} \rightarrow \mathbb{R}^{\geq 0}$ with $f(0)=0$ is super-additive if $f(x+y) \geq f(x)+f(y)$ for all $x, y \geq 0$ and sub-additive if $f(x+y) \leq f(x)+f(y)$ for all $x, y \geq 0$.

Throughout this paper the term sequence will mean a sequence of nonnegative integers and function will mean a real valued function on $\mathbb{R}^{\geq 0}$. Examples of the sequences defined above are $a(n)=\lfloor c n\rfloor$, for any positive real number $c$, which is super-additive, and $a(n)=\lceil c n\rceil$, which is sub-additive. $f(x)=x^{2}$ and $f(x)=\sqrt{x}$ are examples of a super- and a sub-additive function respectively.

As noted in the introduction characteristic sequences of subalgebras $D[x] \subseteq$ $A \subseteq K[x]$ give examples of super-additive sequences. In fact any super-additive sequence arises in this way. If $\{a(n): n=0,1,2, \ldots\}$ is such a sequence and $p \in D$ is a uniformizing element, i.e. $\nu(p)=1$, then the $D$ module spanned by the 
set $\left\{x^{n} / p^{a(n)}: n=0,1,2, \ldots\right\}$ is an algebra because of super-additivity and has $\{a(n): n=0,1,2, \ldots\}$ as a characteristic sequence.

Lemma 2.2. (i) If $\{a(n): n=0,1,2, \ldots\}$ is a nondecreasing super-additive sequence, then $a(\lfloor x\rfloor)$ is a super-additive function.

(ii) If $\{a(n): n=0,1,2, \ldots\}$ is a nondecreasing sub-additive sequence, then $a(\lceil x\rceil)$ is a sub-additive function

(iii) If $f(x)$ is a nondecreasing super-additive function, then $\lfloor f(n)\rfloor$ is a superadditive sequence.

(iv) If $f(x)$ is a nondecreasing sub-additive function, then $\lceil f(n)\rceil$ is a sub-additive sequence.

Proof. If $a(n)$ is super-additive, then

$$
a(\lfloor x+y\rfloor) \geq a(\lfloor x\rfloor+\lfloor y\rfloor) \geq a(\lfloor x\rfloor)+a(\lfloor y\rfloor)
$$

while if it is sub-additive,

$$
a(\lceil x+y\rceil) \leq a(\lceil x\rceil+\lceil y\rceil) \leq a(\lceil x\rceil)+a(\lceil y\rceil) .
$$

If $f(x)$ is super-additive, then

$$
\lfloor f(i+j)\rfloor \geq\lfloor f(i)+f(j)\rfloor \geq\lfloor f(i)\rfloor+\lfloor f(j)\rfloor ;
$$

while if it is sub-additive,

$$
\lceil f(i+j)\rceil \leq\lceil f(i)+f(j)\rceil \leq\lceil f(i)\rceil+\lceil f(j)\rceil .
$$

Lemma 2.3. If $\{a(n): n=0,1,2, \ldots\}$ is a nondecreasing sequence, then $a(\lfloor x\rfloor)$ is an upper semi-continuous function and $a(\lceil x\rceil)$ is a lower semi-continuous function.

Proof. Since if $\left\lfloor x^{\prime}\right\rfloor \leq x^{\prime} \leq x<\left\lfloor x^{\prime}\right\rfloor+1$, then $a(\lfloor x\rfloor)=a\left(\left\lfloor x^{\prime}\right\rfloor\right)$, it follows that $\lim _{x \rightarrow x_{0}^{+}} a(\lfloor x\rfloor)=a\left(\left\lfloor x_{0}\right\rfloor\right)$ and similarly for $\lim _{x \rightarrow x_{0}^{-}} a(\lceil x\rceil)$.

In this paper we are primarily concerned with super-additive sequences. There is a substantial literature concerning super and sub-additive functions, and the previous lemma will allow us to translate those results to the situation in which we are interested.

The next definition follows that in section 2 of 10 :

Definition 2.4. (i) If $\{a(n): n=0,1,2, \ldots\}$ is a nondecreasing sequence, let $\vec{a}(m)=\max \{n: a(n) \leq m\}$ and $\overleftarrow{a}(m)=\min \{n: m \leq a(n)\}$

(ii) If $f(x)$ is a nondecreasing function on $\mathbb{R}^{\geq 0}$, let $\vec{f}(x)=\sup \{y: f(y) \leq x\}$ and $\overleftarrow{f}(x)=\inf \{y: x \leq f(y)\}$.

Proposition 2.5 ([10], Lemma 2.1). (i) If $f$ is lower semi-continuous, then $\vec{f}$ is upper semi-continuous.

(ii) If $f$ is upper semi-continuous, then $\overleftarrow{f}$ is lower semi-continuous.

Proposition 2.6 ([10], Lemma 2.2). (i) If $f$ is lower semi-continuous, then $f=\overleftrightarrow{\leftrightarrows}$.

(ii) If $f$ is upper semi-continuous, then $f=\overleftrightarrow{\rightleftarrows}$.

Proposition 2.7 ([10], Lemma 2.3). (i) If $f$ is lower semi-continuous, then $f$ is sub-additive iff $\vec{f}$ is super-additive.

(ii) If $f$ is upper semi-continuous, then $f$ is super-additive iff $\overleftarrow{f}$ is sub-additive. 
Corollary 2.8. (i) If $\{a(n): n=0,1,2, \ldots\}$ is a nondecreasing super-additive sequence, then $\{\overleftarrow{a}(n): n=0,1,2, \ldots\}$ is a sub-additive sequence.

(ii) If $\{a(n): n=0,1,2, \ldots\}$ is a nondecreasing nondecreasing sub-additive sequence, then $\{\vec{a}(n): n=0,1,2, \ldots\}$ is a super-additive sequence.

(iii) If $\{a(n): n=0,1,2, \ldots\}$ is a nondecreasing sequence, then $a(n)=\overleftrightarrow{\vec{a}}(n)$.

Proof. (i) $a(\lfloor x\rfloor)$ is a super-additive upper semi-continuous function; hence $\overleftarrow{a}(\lfloor x\rfloor)$

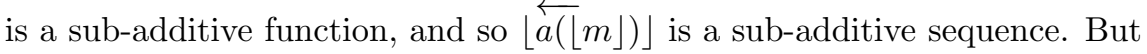

$$
\begin{aligned}
\lfloor\overleftarrow{a}(\lfloor m\rfloor)\rfloor & =\lfloor\inf \{y: m \leq a(\lfloor y\rfloor)\}\rfloor \\
& =\min \{n: m \leq a(n)\} \\
& =\overleftarrow{a}(m)
\end{aligned}
$$

A similar argument proves (ii).

(iii) Clearly

$$
\min \{\ell: a(\ell) \geq a(m)\} \leq m,
$$

which implies

$$
\max \{n: \min \{\ell: a(\ell) \geq n\} \leq m\} \geq a(m)
$$

but if $n>a(m)$ and $a(\ell) \geq n$, then $a(\ell)>a(m)$, and so $\ell>m$, which implies we have equality.

Definition 2.9 ([8). If $\left\{a_{i}(n): n=0,1,2, \ldots\right\}$ for $i=1$ or 2 are nondecreasing sequences, then the shuffle of $a_{1}$ and $a_{2}$, denoted $a_{1} \wedge a_{2}$, is the unique nondecreasing sequence having both $a_{1}$ and $a_{2}$ as disjoint subsequences with every element of $a_{1} \wedge a_{2}$ being in one or the other of these subsequences. Thus there are strictly increasing maps

such that $\phi_{1}\left(\mathbb{Z}^{\geq 0}\right) \cap \phi_{2}\left(\mathbb{Z}^{\geq 0}\right)=\emptyset$,

$$
\phi_{1}, \phi_{2}: \mathbb{Z}^{\geq 0} \rightarrow \mathbb{Z}^{\geq 0}
$$

$$
\phi_{1}\left(\mathbb{Z}^{\geq 0}\right) \cup \phi_{2}\left(\mathbb{Z}^{\geq 0}\right)=\mathbb{Z}^{\geq 0},
$$

and

$$
a_{1} \wedge a_{2}(n)= \begin{cases}a_{1}(m) & \text { if } n=\phi_{1}(m), \\ a_{2}(m) & \text { if } n=\phi_{2}(m),\end{cases}
$$

and such that $\left\{a_{1} \wedge a_{2}(n): n=0,1,2, \ldots\right\}$ is nondecreasing.

The significance of this product is illustrated by the special case of the algebras $\operatorname{Int}\left(E, \mathbb{Z}_{(2)}\right)$ with $E \subseteq \mathbb{Z}_{(2)}$. If $E_{0}$ and $E_{1}$ denote the sets $E \cap 2 \mathbb{Z}_{(2)}$ and $E \cap\left(1+2 \mathbb{Z}_{(2)}\right)$ respectively, then the characteristic sequence of $\operatorname{Int}\left(E, \mathbb{Z}_{(2)}\right)$ is the shuffle product of those of $\operatorname{Int}\left(E_{0}, \mathbb{Z}_{(2)}\right)$ and $\operatorname{Int}\left(E_{1}, \mathbb{Z}_{(2)}\right)$. A similar result holds generally for the intersections of a subset $E$ of $D$ with the various cosets of $\mathcal{M}$ and gives a method for recursively computing the characteristic sequence of $E$ if $E$ is finite, [8], or homogeneous, 9 .

Proposition 2.10. If $\left\{a_{i}(n): n=0,1,2, \ldots\right\}$ for $i=1,2$ are nondecreasing sequences with $a_{1}(0)=a_{2}(0)=0$, then $\left\{\overleftarrow{a_{1} \wedge a_{2}}(n): n=0,1,2, \ldots\right\}=\left\{\overleftarrow{a_{1}}(n)+\right.$ $\left.\overleftarrow{a_{2}}(n): n=0,1,2, \ldots\right\}$

Proof. We proceed by induction on $m$ to show that $\overleftarrow{a_{1} \wedge a_{2}}(m)=\overleftarrow{a_{1}}(m)+\overleftarrow{a_{2}}(m)$ For $m=0$ both sides are 0 . For $m>0$ for any nondecreasing sequence $a(n)$, we have

$$
\overleftarrow{a}(m)=|\{n: a(n)=m-1\}|+\overleftarrow{a}(m-1)
$$


and also, for any nondecreasing sequences $a_{1}(n)$ and $a_{2}(n)$ and any $m>0$,

$$
\left|\left\{n: a_{1} \wedge a_{2}(n)=m-1\right\}\right|=\left|\left\{n: a_{1}(n)=m-1\right\}\right|+\left|\left\{n: a_{2}(n)=m-1\right\}\right| .
$$

Thus

$$
\begin{aligned}
\overleftarrow{a_{1} \wedge a_{2}}(m)= & \left|\left\{n: a_{1} \wedge a_{2}(n)=m-1\right\}\right|+\overleftarrow{a_{1} \wedge a_{2}}(m-1) \\
= & \left|\left\{n: a_{1}(n)=m-1\right\}\right|+\left|\left\{n: a_{2}(n)=m-1\right\}\right| \\
& \quad+\overleftarrow{a_{1}}(m-1)+\overleftarrow{a_{2}}(m-1) \\
= & \overleftarrow{a_{1}}(m)+\overleftarrow{a_{2}}(m) .
\end{aligned}
$$

Corollary 2.11. If $\{a(n): n=0,1,2, \ldots\}$ for $i=1$ or 2 are super-additive sequences, then $\left\{a_{1} \wedge a_{2}(n): n=0,1,2, \ldots\right\}$ is super-additive.

Proof.

$$
a_{1} \wedge a_{2}=\overleftrightarrow{\overleftrightarrow{a_{1} \wedge a_{2}}}=\overleftrightarrow{\overleftarrow{a_{1}}+\overleftrightarrow{a_{1}}}
$$

and the sum of sub-additive sequences is sub-additive.

This description of the shuffle product of sequences translates directly to the case of upper semi-continuous functions:

Definition 2.12. If $f(x)$ and $g(x)$ are upper semi-continuous functions, let $(f \wedge$ $g)(x)=\overleftarrow{\overleftarrow{f(x)}+\overleftrightarrow{g(x)}}$

Corollary 2.13. If $f(x)$ and $g(x)$ are upper semi-continuous super-additive functions, then the function $(f \wedge g)(x)$ is super-additive.

Lemma 2.14. If $\left\{a_{i}(n): n=0,1,2, \ldots\right\}$ for $i=1$ and 2 are nondecreasing sequences with $a_{1}(0)=a_{2}(0)=0$ and $c(n)=a_{1} \wedge a_{2}(n)$, then

$$
c(n)=\max \left\{\min \left(a_{1}(i), a_{2}(j)\right): i+j=n\right\} .
$$

Proof. We proceed by induction, noting first that

$$
\min \left(a_{1}(0), a_{2}(0)\right)=0=c(0)
$$

and

$$
\max \left(\min \left(a_{1}(1), a_{2}(0)\right), \min \left(a_{1}(0), a_{2}(1)\right)\right)=0=c(1) .
$$

If the result holds for $c(n)$, then $\{c(i): i=0, \ldots, n\}$ equals the union of $\left\{a_{1}(i)\right.$ : $i=0, \ldots, k\}$ and $\left\{a_{2}(i): i=0, \ldots, \ell\right\}$ in nondecreasing order for some $k$ and $\ell$ with $(k+1)+(\ell+1)=n+1$ and $a_{1}(k) \leq a_{2}(\ell+1)$ and $a_{2}(\ell) \leq a_{1}(k+1)$. If $i+j=n+1=k+\ell+2$ with $i \leq k$, then $\min \left(a_{1}(i), a_{2}(j)\right)=a_{1}(i)$ since $a_{1}(i) \leq a_{1}(k) \leq a_{2}(\ell+1) \leq a_{2}(j)$; and similarly if $i+j=n+1$ with $j \leq \ell$, then $\min \left(a_{1}(i), a_{2}(j)\right)=a_{2}(j)$. Thus the set $\left\{\min \left(a_{1}(i), a_{2}(j)\right): i+j=n+1\right\}$ contains $\left\{a_{1}(i): i=0, \ldots, k\right\}$ and $\left\{a_{2}(i): i=0, \ldots, \ell\right\}$ and $\min \left(a_{1}(k+1), a_{2}(\ell+1)\right)$, and the maximum of this set is this last element which equals $c(n+1)$.

If $D[x] \subseteq A, B \subseteq K[x]$ are algebras, let $p \in \mathcal{M}$ be such that $\nu(p)=1$ and let $\left\{f_{i}(x) / p^{a(i)}: i=0,1,2, \ldots\right\}$ and $\left.g_{i}(x) / p^{b(i)}: i=0,1,2, \ldots\right\}$ be regular bases (i.e. bases with $f_{i}, g_{i} \in D[x]$ monic of degree $i$ ) for $A$ and $B$ respectively. These exist since the characteristic ideals of $A$ and $B$ are principally generated by powers of $p$. For any $i, j$ we then have $f_{i}(x) g_{j}(x) / p^{\min (a(i), b(j))} \in A \cap B$. Thus in particular if for each $n$ the pair $i, j$ is chosen so that $i+j=n$ and $\min (a(i), b(j))$ is largest and this value is denoted $c(n)$ and $h_{n}(x)=f_{i}(x) g_{j}(x)$, then $h_{n}(x) / p^{c(n)} \in A \cap B$, 
and by the previous lemma $c(n)=a \wedge b(n)$. Thus the shuffle of the characteristic sequences of $A$ and $B$ gives a lower bound for the characteristic sequence of $A \cap B$.

\section{S-PERIODICITY}

Definition 3.1. A nondecreasing sequence $\{a(n): n=0,1,2, \ldots\}$ with $a(0)=0$ will be called $S$-periodic if there are positive integers $q$ and $h$ such that

$$
a(n+q)=a(n)+h
$$

for all $n \geq 0$. The integer $q$ will be referred to as the period of the sequence and $h$ as the height. It is not assumed that the period, $q$, is the smallest positive integer for which this condition holds. That integer will be referred to as the minimal period.

Example 3.2. The sequence $\{h \cdot\lfloor n / q\rfloor: n=0,1,2, \ldots\}$ is $S$-periodic of period $q$ and height $h$. More generally if $q>r_{1} \geq r_{2} \geq \cdots \geq r_{h} \geq 0$ and $q>s_{h} \geq s_{h-1} \geq$ $\cdots \geq s_{1} \geq 0$ are integers, then the sequences $\left\{\sum_{i=1}^{h}\left\lfloor\frac{n+r_{i}}{q}\right\rfloor: n=0,1,2, \ldots\right\}$ and $\left\{\sum_{i=1}^{h}\left\lceil\frac{n-s_{i}}{q}\right\rceil: n=0,1,2, \ldots\right\}$ are $S$-periodic of period $q$ and height $h$.

The name $S$-periodic (step periodic) is chosen to reflect that fact that such a sequence can be expressed as $h \cdot\lfloor n / q\rfloor+a(n \bmod q)$, i.e., the sum of a step sequence and a periodic one.

Lemma 3.3. If $\{a(n): n=0,1,2, \ldots\}$ is a nondecreasing $S$-periodic sequence with period $q$ and height $h$, then the sequences $\overleftarrow{a}(n)$ and $\vec{a}(n)$ are $S$-periodic of period $h$ and height $q$.

Proof.

$$
\begin{aligned}
\overleftarrow{a}(m) & =\min \{n: m \leq a(n)\} \\
& =\min \{n: m \leq a(n+q)-h\} \\
& =\min \{n: h+m \leq a(n+q)\} \\
& =\min \left\{n^{\prime}-q: h+m \leq a\left(n^{\prime}\right)\right\} \\
& =\min \left\{n^{\prime}: h+m \leq a\left(n^{\prime}\right)\right\}-q \\
& =\overleftarrow{a}(m+h)-q .
\end{aligned}
$$

The calculation for $\vec{a}(n)$ is similar.

Proposition 3.4. If $\{a(n): n=0,1,2, \ldots\}$ is a nondecreasing $S$-periodic sequence of period $q$ and height $h$ and $a(0)=0$, then

$$
a(n)=\sum_{i=1}^{h}\left\lfloor\frac{n+r_{i}}{q}\right\rfloor=\sum_{i=1}^{h}\left\lceil\frac{n-s_{i}}{q}\right\rceil
$$

with $r_{i}=q-\overleftarrow{a}(i)$ and $s_{i}=\vec{a}(i)$

Proof. The sequence $\left\{\sum_{i=1}^{h}\left\lfloor\frac{n+r_{i}}{q}\right\rfloor: n=0,1,2, \ldots\right\}$ is $S$-periodic of period $q$ and height $h$; hence it suffices to check that it agrees with $\{a(n): n=0,1,2, \ldots\}$ for $0 \leq n \leq q$. If the $r_{i}$ 's are as in the statement of the proposition so that 
$q>r_{1} \geq r_{2} \geq \cdots \geq r_{h} \geq 0$, then for each $0<j \leq h$ we have $a(n)=j$ if $q-r_{j} \leq n<q-r_{j+1}$ and $a(n)=0$ if $n<q-r_{1}$. If $q-r_{j} \leq n<q-r_{j+1}$, then

$$
\left\lfloor\frac{n+r_{i}}{q}\right\rfloor= \begin{cases}1 & \text { if } i \leq j \\ 0 & \text { if } i>j\end{cases}
$$

and so $\sum_{i=1}^{h}\left\lfloor\frac{n+r_{i}}{q}\right\rfloor=j=a(n)$ as required. The proof of the second equality is similar.

Note that since $\left\lfloor\frac{n}{q}\right\rfloor=\sum_{i=0}^{k-1}\left\lfloor\frac{n+i q}{k q}\right\rfloor$, this representation does not require $q$ to be the minimal period and that by replacing $q$ by a multiple it may be assumed that the period of $a(n)$ is as large as desired.

Corollary 3.5. (i) The S-periodic sequence

$$
\left\{\sum_{1}^{h}\left\lfloor\frac{n+r_{i}}{q}\right\rfloor: n=0,1,2, \ldots\right\}
$$

is super-additive if and only if the $r_{i}$ 's satisfy the inequalities

$$
\left(q-r_{i}\right)+\left(q-r_{j}\right) \geq\left(q-r_{i+j}\right) \quad \text { if } i+j<h
$$

and

$$
\left(q-r_{i}\right)+\left(q-r_{j}\right) \geq\left(2 q-r_{i+j-h}\right) \quad \text { if } h \leq i+j .
$$

(ii) The $S$-periodic sequence

$$
\left\{\sum_{1}^{h}\left\lceil\frac{n-s_{i}}{q}\right\rceil: n=0,1,2, \ldots\right\}
$$

is sub-additive if and only if the $s_{i}$ 's satisfy the inequalities

$$
s_{i}+s_{j} \leq\left(s_{i+j}\right) \quad \text { if } i+j<h
$$

and

$$
s_{i}+s_{j} \leq\left(q+s_{i+j-h}\right) \text { if } h \leq i+j .
$$

Proof. $\{a(n): n=0,1,2, \ldots\}$ is super-additive if and only if $\{\overleftarrow{a}(n): n=$ $0,1,2, \ldots\}$ is sub-additive and is sub-additive if and only if $\{\vec{a}(n): n=0,1,2, \ldots\}$ is super-additive.

Proposition 3.6. If $\left\{a_{i}(n): n=0,1,2, \ldots\right\}$ for $i=1$ or 2 are $S$-periodic sequences of periods $q_{1}$ and $q_{2}$ and heights $h_{1}$ and $h_{2}$, then $\left\{\left(a_{1}+a_{2}\right)(n): n=0,1,2, \ldots\right\}$ is $S$-periodic of period lcm $\left(q_{1}, q_{2}\right)$ and height $\left(h_{1} q_{2}+h_{2} q_{1}\right) / \operatorname{gcd}\left(q_{1}, q_{2}\right)$.

Proof. If $q_{1}=q_{2}=q$, then clearly $a_{1}+a_{2}(n)$ is $S$-periodic of period $q$ and height $h_{1}+h_{2}$. Since $a_{1}(n)$ is periodic of period $q_{1}$, it is also periodic of period $k_{1} q_{1}$ and height $k_{1} h_{1}$ for any $k_{1}$. Similarly for $a_{2}(n)$. Thus taking $k_{1}=q_{2} / \operatorname{gcd}\left(q_{1}, q_{2}\right)$ and $k_{2}=q_{1} / \operatorname{gcd}\left(q_{1}, q_{2}\right)$, we obtain the result.

Proposition 3.7 (4, Proof of Theorem 3.1). If $\left\{a_{i}(n): n=0,1,2, \ldots\right\}$ for $i=1$ or 2 are $S$-periodic sequences of periods $q_{1}$ and $q_{2}$ and heights $h_{1}$ and $h_{2}$, then $\left\{a_{1} \wedge a_{2}(n): n=0,1,2, \ldots\right\}$ is $S$-periodic of period $\left(q_{1} h_{2}+q_{2} h_{1}\right) / \operatorname{gcd}\left(h_{1}, h_{2}\right)$ and height lcm $\left(h_{1}, h_{2}\right)$. 
Proof. Since

$$
\overleftarrow{a_{1} \wedge a_{2}}=\overleftarrow{a_{1}}+\overleftarrow{a_{2}}
$$

the result follows from the previous proposition.

The finite sums $\sum_{i=1}^{\ell}\left\lfloor\frac{x}{q_{i}}\right\rfloor$ also give examples of $S$-periodic super-additive sequences. In fact:

Proposition 3.8. If $\ell$ is finite and $q_{1} \leq q_{2} \leq \cdots \leq q_{\ell}$ is a nondecreasing list and $a(n)=\sum_{i=1}^{\ell}\left\lfloor\frac{n}{q_{i}}\right\rfloor$, then $\{a(n): n=0,1,2, \ldots\}$ is an $S$-periodic super-additive sequence of period $Q=\operatorname{lcm}\left(\left\{q_{i}\right\}_{i=1}^{\ell}\right)$ and height $\sum_{j=1}^{\ell} Q / q_{j}$.

Proof. Let $Q_{j}=Q / q_{j}$. We, then have

$$
\left\lfloor\frac{n}{q_{i}}\right\rfloor=\sum_{j=0}^{Q_{j}-1}\left\lfloor\frac{n+j q_{i}}{Q}\right\rfloor ;
$$

hence

$$
a(n)=\sum_{i=1}^{\ell} \sum_{i=0}^{Q_{j}-1}\left\lfloor\frac{n+j q_{i}}{Q}\right\rfloor .
$$

It is not the case that any $S$-periodic super-additive sequence can be represented as a finite $\operatorname{sum} \sum_{i=1}^{\ell}\left\lfloor\frac{n}{q_{i}}\right\rfloor$ however.

Example 3.9. The sequence $\{a(n): n=0,1,2, \ldots\}$ with $a(n)=\left\lfloor\frac{n+3}{5}\right\rfloor+$ $\left\lfloor\frac{n+2}{5}\right\rfloor+\left\lfloor\frac{n}{5}\right\rfloor+\left\lfloor\frac{n}{5}\right\rfloor$ is super-additive since the values $q=5, r_{1}=3, r_{2}=2$, $r_{3}=0, r_{4}=0$ satisfy the inequalities of Corollary 3.5 . If this sequence were equal to $\sum_{i=1}^{\ell}\left\lfloor\frac{n}{q_{i}}\right\rfloor$, then each $q_{i}$ would be a divisor of 5 and so, since $a(1)=0$, would equal 5. This would imply $a(n)=0$ for $n<5$; however $a(2)=1$.

\section{Generators}

Definition 4.1. If $\{a(n): n=0,1,2, \ldots\}$ is a super-additive sequence, then a positive integer $g$ will be called a generator of $a$ if $a(i)+a(j)<a(g)$ for all $i, j$ positive integers with $i+j=g$ and 1 will be called a generator if $a(1)>0$.

Example 4.2. If $a(n)=\nu_{p}(n !)$ is the $p$-adic norm with respect to a fixed prime, $p$, of $n$ !, then $a(n)$ is super-additive since if $i+j=n$, then $i ! j$ ! divides $n$ !. The generators for this sequence are $p^{k}$ for $k=1,2, \ldots$ since $p$ divides $p^{k} ! / i ! j$ ! if $i+j=p^{k}$ with $i, j>0$, and if $p^{k}>r>0$, then $p$ does not divide $\left(p^{k}+r\right) ! / p^{k} ! r$.

Proposition 4.3. If $\{a(n): n=0,1,2, \ldots\}$ is the characteristic sequence of an algebra $D[x] \subseteq A \subseteq K[x]$ and $G$ is the set of generators of this sequence and if $H=$ $\left\{h_{n}(x) / p^{a(n)}: n=0,1,2, \ldots\right\}$ is a regular basis for $A$, then the set $\left\{h_{g}(x) / p^{a(g)}\right.$ : $g \in G\}$ is a multiplicative generating set for the algebra A. 
Proof. It suffices to show that each $h_{n}(x) / p^{a(n)}$ is in the algebra generated by the set $\left\{h_{g}(x) / p^{a(g)}: g \in G\right\}$. We do this by induction on $n$. If $n \in G$ there is nothing to prove. If $n \notin G$, then $a(n)=a(i)+a(j)$ for some $i, j>0$, and so the leading coefficient of $h_{i}(x) h_{j}(x) / p^{a(i)+a(j)}$ is a $D$ unit multiple of that of $h_{n}(x)$; i.e. there exists a unit $u \in D$ such that $h_{n}(x) / p^{a(n)}-u h_{i}(x) h_{j}(x) / p^{a(i)+a(j)}$ is of degree less than $n$. By induction this implies that $h_{n}(x) / p^{n}$ is in the algebra generated by $\left\{h_{g}(x) / p^{a(n)}: g \in G\right\}$ as required.

Example 4.4. The algebra $\operatorname{Int}\left(\mathbb{Z}, \mathbb{Z}_{(p)}\right)$ has as a regular $\mathbb{Z}_{(p)}$ basis the binomial polynomials $\left(\begin{array}{l}x \\ n\end{array}\right)$. Since the $n$-th of these is the quotient of a monic polynomial by $n$ !, the characteristic sequence is that given in the previous example and the generators are as given there. From this it follows that the Fermat polynomials defined recursively by $f_{0}(x)=x, f_{i+1}(x)=\left(f_{i}^{p}(x)-f_{i}(x)\right) / p$ also form a generating set over $\mathbb{Z}_{(p)}$ for $\operatorname{Int}\left(\mathbb{Z}, \mathbb{Z}_{(p)}\right)$. Since this algebra occurs in algebraic topology as the $K$-homology of $\mathbb{C} P^{\infty}([7])$, this fact has been of use in homological algebra calculations concerning that space.

While the previous proposition justifies the choice of the term generator for a super-additive sequence, it is still somewhat misleading since it implies that a sequence $a(n)$ can be recovered from its list of generators or that an arbitrary increasing list determines a unique sequence. For these to hold we must also retain the multiplicities of the generators:

Definition 4.5. If $\{a(n): n=0,1,2, \ldots\}$ is super-additive sequence and $g$ a generator of $a$, then the multiplicity of $g$ is the integer $e$ given by

$$
e=a(g)-\max \{a(i)+a(j): i+j=g, i, j>0\} .
$$

If $g=1$ is a generator, then its multiplicity is $a(1)$.

Proposition 4.6. If $\left\{\left(g_{i}, e_{i}\right): i=1,2, \ldots\right\}$ is a set of pairs of positive integers with $g_{i+1}>g_{i}$ for all $i$, then there is a unique super-additive sequence $a(n)$ with generators $\left\{g_{i}\right\}$ of multiplicities $\left\{e_{i}\right\}$.

Proof. Define $a(n)$ recursively by $a(n)=0$ for $n<g_{1}$ and

$$
a(n)= \begin{cases}\max \{a(i)+a(j): i+j=n, i, j>0\} & \text { if } n \neq g_{i} \\ \max \{a(i)+a(j): i+j=n, i, j>0\}+e_{i} & \text { if } n=g_{i} .\end{cases}
$$

If $\{a(n): n=0,1,2, \ldots\}$ is a super-additive sequence, then the relation between the generators of $a(n)$ and the values of the sub-additive sequence $\overleftarrow{a}(n)$ is given in the following proposition:

Proposition 4.7. If $\{a(n): n=0,1,2, \ldots\}$ is a super-additive sequence, then the generators are those values of the sequence $\{\overleftarrow{a}(n): n=0,1,2, \ldots\}$ with the property that

$$
\overleftarrow{a}(n)<\overleftarrow{a}(k)+\overleftarrow{a}(\ell)
$$

if $k+\ell=n$ and $k, \ell>0$.

Proof. First note that if $a(n)=a(n-1)$, then $n$ cannot be a generator of $a$. Since $a(n) \leq a(n-1)+a(1)$, then $a(n)=a(n-1)+a(1)$ since $a$ is super-additive. Next note that if $a(n-1)<a(n)$, then $n$ is a value of $\overleftarrow{a}$, since in this case $\overleftarrow{a}(a(n))=n$ If $n$ is not a generator, then $a(n)=a(i)+a(j)$ for some $i, j>0$ with $i+j=n$. By 
decreasing $i, j$ if necessary we may assume that $a(n)=a(i)+a(j)$, that $i+j \leq n$ and that $a(i-1)<a(i)$ and $a(j-1)<a(j)$. Thus

$$
i=\overleftarrow{a}(a(i))
$$

and

$$
j=\overleftarrow{a}(a(j))
$$

and so

$$
n=\overleftarrow{a}(a(n)) \geq \overleftarrow{a}(a(i))+\overleftarrow{a}(a(j))=i+j
$$

Since $a$ is super-additive, $\overleftarrow{a}$ is sub-additive; hence

$$
n=\overleftarrow{a}(a(i))+\overleftarrow{a}(a(j))
$$

Thus the generators of $a$ are among the values of $\overleftarrow{a}$ satisfying the condition in the statement of the proposition.

Conversely, suppose that $m=\overleftarrow{a}(n)$ is a value of $\overleftarrow{a}$ and that $\overleftarrow{a}(n)=m=$ $\overleftarrow{a}(k)+\overleftarrow{a}(\ell)$ for some $k \cdot \ell>0$ with $k+\ell=n$. It need not be the case that either $k$ or $\ell$ is a value of $a$. However, there exist $k^{\prime} \geq k$ and $\ell^{\prime} \geq \ell$ such that $\overleftarrow{a}\left(k^{\prime}\right)=\overleftarrow{a}(k)$ and $\overleftarrow{a}\left(\ell^{\prime}\right)=\overleftarrow{a}(\ell)$ and $k^{\prime}=a(i)$ and $\ell^{\prime}=a(j)$. Since $k^{\prime}, \ell^{\prime}>0$ it follows that $i, j>0$ also. We thus have

$$
m=\overleftarrow{a}(a(m))=\overleftarrow{a}(n)=\overleftarrow{a}(a(i))+\overleftarrow{a}(a(j))=i+j
$$

and

$$
a(m)=n \leq k^{\prime}+\ell^{\prime}=a(i)+a(j) .
$$

Since $a$ is superadditive, equality must hold in the last equation, and so $m$ is not a generator of $a$.

Corollary 4.8. If $\left\{a_{i}(n): n=0,1,2, \ldots\right\}$ for $i=1$ or 2 are super-additive sequences, then the generators of $a_{1} \wedge a_{2}$ are the values of $\overleftarrow{a}_{1}+\overleftarrow{a}_{2}$ satisfying the condition

$$
\left(\overleftarrow{a}_{1}+\overleftarrow{a}_{2}\right)(n)<\left(\overleftarrow{a}_{1}+\overleftarrow{a}_{2}\right)(k)+\left(\overleftarrow{a}_{1}+\overleftarrow{a}_{2}\right)(\ell) \text { if } k+\ell=n, \quad k, \ell>0
$$

Definition 4.9. A sequence is finitely generated if its list of generators is finite.

Proposition 4.10. If $\{a(n): n=0,1,2, \ldots\}$ is $S$-periodic, then it is finitely generated.

Proof. Since $a(n+q)=a(n)+h=a(n)+a(q), a$ cannot have a generator of size $>q$.

It is not the case that all finitely generated sequences are $S$-periodic.

Example 4.11. The sequence $a(0)=a(1)=0, a(2)=a(3)=2, a(4)=4$, $a(n)=n$ for $n \geq 5$ is generated by $\{(2,2),(5,1)\}$ but is clearly not $S$-periodic.

Proposition 4.12. If $\{a(n): n=0,1,2, \ldots\}$ is a finitely generated super-additive sequence with generators $g_{1} \ldots g_{\ell}$ and $a_{i}=a\left(g_{i}\right)$ for $i=1, \ldots, \ell$, then there exists an integer $N$ and an $S$-periodic sequence $b(n)$ such that $b(n)=a(n)$ for $n>N$. The minimal period of $b(n)$ divides $g_{i}$ for each index $i$ for which $a_{i} / g_{i}$ is maximal. 
Proof. Let $g_{k}$ be one of the generators $g_{i}$ for which $a_{i} / g_{i}$ is maximal. For any $n$ larger than the largest of the $g_{i}$ 's we have

$$
\begin{aligned}
a(n) & =\max \{a(i)+a(j): i+j=n, i, j>0\} \\
& =\max \left\{\sum_{i=1}^{\ell} c_{i} a_{i}: \sum_{i=1}^{\ell} c_{i} g_{i} \leq n, c_{i} \geq 0\right\} .
\end{aligned}
$$

If $j \neq k$ and $c_{j} \geq g_{k}$, then

$$
c_{j} g_{j}+c_{k} g_{k}=\left(c_{j}-g_{k}\right) g_{j}+\left(c_{k}+g_{j}\right) g_{k}
$$

and

since $g_{j} a_{k} \geq g_{k} a_{j}$. Thus

$$
c_{j} a_{j}+c_{k} a_{k} \leq\left(c_{j}-g_{k}\right) a_{j}+\left(c_{k}+g_{j}\right) g_{k}
$$

$$
a(n)=\max \left\{\sum_{i=1}^{\ell} c_{i} a_{i}: \sum_{i=1}^{\ell} c_{i} g_{i} \leq n, 0 \leq c_{i}<g_{k} \text { if } i \neq k\right\},
$$

and, if $n$ is large enough $\left(n>N=g_{k} \sum_{i=0}^{\ell} g_{i}\right)$, then we also may assume $c_{k}>0$ :

$$
a(n)=\max \left\{\sum_{i=1}^{\ell} c_{i} a_{i}: \sum_{i=1}^{\ell} c_{i} g_{i} \leq n, c_{i}<g_{k} \text { if } i \neq k \text { and } c_{k}>0\right\} .
$$

The set of coefficients over which this maximum is taken,

$$
\mathcal{C}_{1}=\left\{\left(c_{1} \ldots c_{\ell}\right): \sum_{i=1}^{\ell} c_{i} g_{i} \leq n, c_{i}<g_{k} \text { if } i \neq k \text { and } c_{k} \geq 0\right\},
$$

is in $1-1$ correspondence with the set

$$
\mathcal{C}_{2}=\left\{\left(c_{1} \ldots c_{\ell}\right): \sum_{i=1}^{\ell} c_{i} g_{i}=n+g_{k},, 0 \leq c_{i}<g_{k} \text { if } i \neq k \text { and } c_{k}>0\right\}
$$

via $\left(c_{1} \ldots c_{\ell}\right)$ corresponding with $\left(c_{1} \ldots c_{k}+1, \ldots, c_{\ell}\right)$.

Since $a(n)=\max \left\{\sum_{i=1}^{\ell} c_{i} a_{i}:\left(c_{1} \ldots c_{\ell}\right) \in \mathcal{C}_{1}\right\}$ we have

$$
\begin{aligned}
a\left(n+g_{k}\right) & =\max \left\{\sum_{i=1}^{\ell} c_{i} a_{i}:\left(c_{1} \ldots c_{\ell}\right) \in \mathcal{C}_{2}\right\} \\
& =a_{k}+\max \left\{\sum c_{i} a_{i}:\left(c_{1} \ldots c_{\ell}\right) \in \mathcal{C}_{1}\right\} \\
& =a_{k}+a(n) .
\end{aligned}
$$

Given $n \geq 0$, choose $r$ such that $n+r g_{k}>N$ and define $b(n)=a\left(n+r g_{k}\right)-r a_{k}$. The previous calculation shows that this definition is independent of the choice of $r$ and $b(n)=a(n)$ if $n>N$. Also if $i+j=n$ and $r, s$ are such that $r i>N$ and $s j>N$, then $b(i)+b(j)=a\left(i+r g_{k}\right)-r a_{k}+a\left(j+s g_{k}\right)-s a_{k} \leq a\left(i+r g_{k}+j+s g_{k}\right)-(r+s) a_{k}=$ $b(n)$. Thus $\{b(n): n=0,1,2, \ldots\}$ is super-additive, $S$-periodic with period dividing $g_{k}$, and agrees with $\{a(n): n=0,1,2, \ldots\}$ for $n$ sufficiently large.

Corollary 4.13. If $\{a(n): n=0,1,2, \ldots\}$ is a finitely generated super-additive sequence with generators $g_{1}<g_{2}<\cdots<g_{\ell}$ and $a(n)$ is $S$-periodic, then $a\left(g_{\ell}\right) / g_{\ell}>$ $a\left(g_{i}\right) / g_{i}$ for all $i<\ell$. 
Proof. Since $g_{\ell}$ is a generator, $a\left(g_{\ell}\right) / g_{\ell} \neq a\left(g_{i}\right) / g_{i}$ for any $g_{i}$ dividing $g_{\ell}$. Thus if $a\left(g_{\ell}\right) / g_{\ell}=a\left(g_{i}\right) / g_{i}$ for some $i<\ell$, then $a(n)$ has a minimal period dividing both $g_{\ell}$ and $g_{i}$, hence smaller than either. But no $S$-periodic sequence has a generator larger than its minimal period.

Remark 4.14. This corollary gives a necessary condition for a finitely generated sequence to be $S$-periodic which is not sufficient. If $a(n)$ is the sequence with generators 2 and 5 of multiplicities 3 and 2 , then $a(2)=3, a(5)=8$ and so $a(2) / 2=3 / 2<8 / 5=a(5) / 5$. The sequence $a(n)$ begins with $0,0,3,3,6,8,9$; hence it is not $S$-periodic since $9=a(6) \neq 8+0=a(5)+a(1)$.

Proposition 4.15. If $a(n)$ is a super-additive sequence which is asymptotically $S$-periodic, i.e. there exists an $S$-periodic sequence $b(n)$ such that $a(n)=b(n)$ for $n \geq N$, then $a(n)$ is finitely generated.

Proof. Since $b(n)$ is $S$-periodic it has a representation of the form

$$
b(n)=\sum_{i=0}^{h-1}\left\lfloor\frac{n+r_{i}}{q}\right\rfloor,
$$

and we may assume, by replacing $q$ by a multiple if necessary, that $q>N$. Note that since $b(n)$ is an $S$-periodic function it has the property $b(t q+s)=t h+b(s)=b(t q)+$ $b(s)$. Also note that if the result holds in the special case $a(n)=0$ for $n<q$, then it will hold in general. We claim that in this case $a(n)$ is generated by a generator $q$ of multiplicity $h$ together with generators $\left\{q+r_{i}: r_{i}>0\right\}$, each of multiplicity 1. Let $c(n)$ be the sequence with these generators. We proceed by induction on $n$. It is clear that $c(n)=0$ for $n<q$ and $c(n)=b(n)=a(n)$ for $n \leq 2 q$. Suppose that $c(n)=b(n)$ for $n<m q+s$ with $0<s \leq q$ and consider $c(m q+s)$. We have $b(m q+s)=b((m-1) q+(m+s))=b((m-1) q)+b(m+s)=c((m-1) q)+c(m+s)$. Thus $c(m q+s) \geq b(m q+s)$. On the other hand, if $i+j=m q+s$ with $i, j \geq q$, then $c(i)+c(j)=b(i)+b(j) \leq b(m q+s)$ since $b(n)$ is super-additive; while if $i+j=m q+s$ with $0<i<q$, then $c(i)+c(j)=c(j)=b(j) \leq b(m q+s)$ since $b(n)$ is nondecreasing. Thus $c(m q+s) \leq b(m q+s)$.

Remark 4.16. It should be noted that the hypothesis in this proposition is not the same as requiring that for some positive integers $q$ and $h$ we have $a(n+q)=a(n)+h$ for all $n>N$. This weaker condition is satisfied by the super-additive sequence $a(0)=0, a(n)=n-1$ for $n>0$, which is not finitely generated since $a(i)+a(j)=$ $i+j-2=a(i+j)-1$ for all $i, j>0$.

\section{REFERENCES}

[1] M. Bhargava, P-orderings and polynomial functions on arbitrary subsets of Dedekind rings, J. Reine Angew. Math. 490 (1997), 101-127. MR1468927 (98j:13016)

[2] _ The factorial function and generalizations, Am. Math. Monthly 107 (2000), 783799. MR.1792411(2002d:05002)

[3] _ On P-orderings, rings of integer valued polynomials and ultrametric analysis, Journal of the Amer. Math. Soc. 22(4) (2009), 963-993. MR2525777(2010i:13022)

[4] M. Bhargava, P.-J. Cahen, and J. Yermanian, Finite generation properties for various rings of integer valued polynomials, Journal of Algebra 322 (2009), 1129 -1150. MR2537676

[5] J. Boulanger and J.-L. Chabert, Asymptotic behavior of characteristic sequences of integervalued polynomials, J. Number Theory 80 (2000), 238-259. MR1740513 (2001g:13043)

[6] P.-J. Cahen and J.-L. Chabert, Integer Valued Polynomials, Amer. Math. Soc., Providence, R.I., 1997. MR.1421321 (98a:13002) 
[7] F. Clarke, Self maps of BU, Math. Proc. Cam. Phil. Soc. 89 (1981), 491-500. MR602302 (82f:55007)

[8] K. Johnson, P-orderings of finite subsets of Dedekind domains, J. Algebraic Combinatorics 30 (2009), 233-253. MR2525060 (2010g:13030)

[9] Limits of characteristic sequences of integer-valued polynomials on homogeneous sets, J. Number Theory 129 (2009), 2933-2942. MR2560844 (2010j:11048)

[10] L.S. Osterdal, Sub-additive functions and their (pseudo-) inverses, J. Math. Anal. Appl. 317 (2006), 724 -731. MR2209592 (2006k:26010)

[11] A. Ostrowski, Uber Ganzwertige Polynome in Algebraischen Zahlkorper, J. Reine Angew. Math. (Crelle) 149 (1919), 117-124.

[12] G. Polya, Uber Ganzwertige Polynome in Algebraischen Zahlkorper, J. Reine Angew. Math. (Crelle) 149 (1919), 97-116.

Department of Mathematics, Dalhousie University, Halifax, Nova Scotia, B3H 4R2, CANADA

E-mail address: johnson@mathstat.dal.ca 